(Aus dem physiologischen Institut der Universität Breslau.)

\title{
Die Prüfung der Manometer mit Druckschwankungen von bekannter Form.
}

Von

\section{K. Hürthle.}

(Mit 3 Textfiguren und Tafel III.)

Nachdem in der vorhergehenden Abhandlung nachgewiesen worden ist, dass der Gültigkeitsbereich der $\mathrm{Mach}$ 'schen Theorie in ihrer von Frank auf die elastischen Manometer übertragenen Form ein beschränkter ist und bei den für die Untersuchung des Blutdrucks geeigneten Konstruktionen seine Grenze hat, ist auch die auf die Theorie gegründete Prüfungsmethode mit Hilfe der Eigenschwingungen der Manometer nicht mehr von allgemeiner Bedeutung, sondern auf den Gültigkeitsbereich der Theorie beschränkt. Denn es ist nicht anzunehmen und würde erst noch eines besonderen Nachweises bedürfen, dass Schwingungszahl und Dekrement auch in den Fällen ein Masstab für die Güte der Manometer sind, in welchen die Theorie nicht mehr anwendbar ist; so lässt sich z. B. von vornherein nicht entscheiden, ob die Leistungsfähigkeit eines Manometers durch Verkleinerung der wirksamen Masse (Verkürzung der Zuleitungsröhre) auch in dem Falle erhöht wird oder nicht, wenn diese Änderung ohne Einfluss auf die Schwingungszahl ist (Beispiele s. die Abschnitte $\mathrm{E}$ und $\mathrm{F}$ der vorhergehenden Abhandlung). Desgleichen lässt sich über die Leistungen des Kapillarmanometers auf Grund der Theorie nichts Sicheres aussagen (s. Abschnitt D der vorhergehenden Abhandlung). Das Anwendungsgebiet der Schwingungsmethode ist also ein beschränktes.

Aber auch innerhalb des Bereiches der Theorie stehen der Schwingungsmethode verschiedene Bedenken entgegen:

Prinzipiell unzulässig ist die Anwendung der Grundgleichung $M \frac{d^{2} x}{d t^{2}}+E x+K \frac{d x}{d t}=\sum_{n=0}^{n}\{A \cos (n t+\tau)\} \ldots$ zur Korrektur der Pflüger's Archiv für Physiologie. Bd. 18 i. 
Kurven, weil das Glied $\mathbf{\Sigma}$..., welches die zu registrierende Kraft (den Blutdruck) darstellt, unter dem Einfluss der Manometerbewegung selbst verändert wird, und die Grösse der Veränderung sich der Berechnung entzieht. Aus diesem Grunde kann die Schwankung der ursprünglichen Kraft aus der registrierten Kurve überhaupt nicht genau ermittelt werden.

Eine weitere Fehlerquelle für die Korrektur der Kurven nach der Sehwingungsmethode besteht in der Inkonstanz der Elastizitätskonstanten bei wechselndem Druck, die bei manchen Instrumenten, z. B. bei dem von Frank verwandten Membranmanometer, so bedeutend ist, dass $\boldsymbol{E}^{\prime}$ fast um das Vierfache schwankt ${ }^{1}$ ). Diese Inkonstanz hat zur Folge, dass Schwingungszahl und Dekrement eines solchen Instrumentes bei gleichbleibender wirksamer Masse mit dem Druck wechseln. Die Korrektur auf Grund eines Schwingungsversuches gilt daher nur für einen Druckbezirk, dessen Grenzen mit der Art des Manometers wechseln.

Weiterbin hat die Anwendung der Schwingungsmethode ein konstantes Dekrement und Proportionalität zwischen Volumverschiebung. und Ordinatenhöhe zur Voraussetzung. Beide Voraussetzungen sind aber in Wirklichkeit selten erfültt (vgl. S. 180).

Zieht man schliesslich in Betracht, dass zur Korrektur der Kurven mit Hilfe der Schwingungsmethode die Berechnung des zweiten Differentialquotienten erforderlich ist, die bekanntlich bezüglich der Genauigkeit der Resultate eine sehr missliche Sache ist, so kommt man zu dem Ergebnis, dass die mathematische Korrektur der Kurven nicht allein der mathematisehen Genauigkeit entbehrt, sondern auch desjenigen Grades der praktischen, welche von einer Methode gefordert werden muss, die Anspruch darauf macht, den wahren Druckverlauf festzustellen.

Sieht man sich nun nach geeigneteren Methoden zur Prüfung der Manometer um, so kommen in Betracht:

1. Die Ermittelung der Einstell ung s zeit $^{2}$ ) des aperiodischen Manometers; diese halte ich trotz des absprechenden Urteils von Frank ${ }^{3}$ ) auch heute noch für ein sehr wertvolles Kriterium der Leistungsfähigkeit der Manometer.

1) Frank, Kritik S. 511.

2) Siehe die vorhergehende Abhandlung S. 204.

3) Frank, Prinzipien ușw. Zeitschr. f. Biol. Bd. 53 S. 452. 
Die Prüfung der Manometer mit Druckschwankungen von bekannter Form. 227

2. Die Vergleichung der registrierten Kurven mit denjenigen eines Instrumentes von nachweisbar grösserer Leistungsfähigkeit. Diese früher von mir benutzte Methode ${ }^{1}$ ) hat auch $\mathrm{Frank}^{2}$ ) neuerdings empfohlen.

3. Eingehender soll hier die Methode der Prüfung mit künstlichen Druckschwankungen von bekannter Form

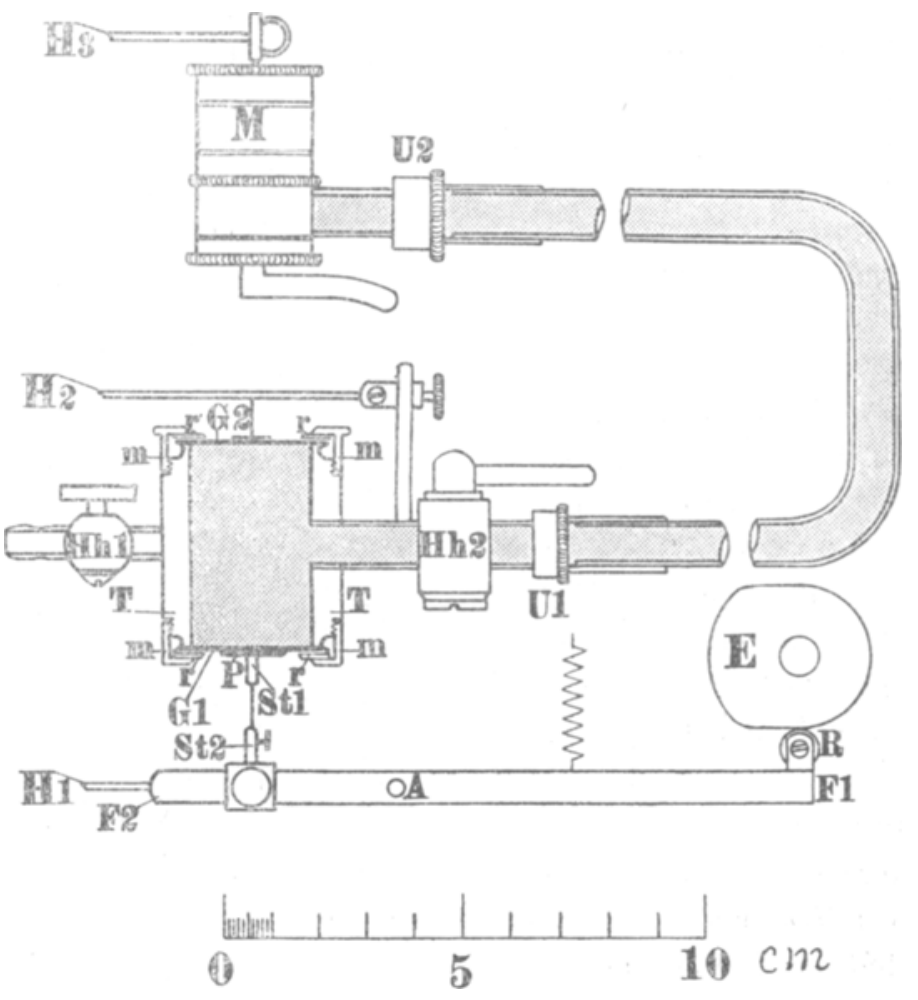

Fig. 1 .

behandelt werden, welche von Fick und Mach benutzt, von Donders speziell für die Prüfung der Lufttransmissionssphygmographen eingerichtet und von mir $^{3}$ ) zum Vergleich der Manometer

1) K. Hürthle, Experimentelle Prüfung usw. Pflüger's Arch. Bd. 47 S. 14. 1890. - K. Hürthle, Vergleichende Prüfung usw. Pflüger's Arch. Bd. 55 S. 336.1894.

2) Frank, Prinzipien usw. Zeitschr. f. Biol. Bd. 53 S. 452 und 453.

3) K. Hürthle, Vergleichende Prüfung usw. Pflüger's Arch. Bd. 55 S. 322. 
mit Wasser- und Lufttransport gebraucht worden ist ${ }^{1}$ ). Das von mir beschriebene Verfahren halte ich trotz der absprechenden Kritik Frank's'), die keinerlei sachliche Fehler nachweist, auch heute noch für geeignet; ich habe es aber noch dahin erweitert, dass mittels desselben nicht allein die Übereinstimmung der vom Manometer gezeichneten Kurve mit der künstlich erzeugten Druckschwankung, sondern auch die Rückwirkung des Manometers auf die einwirkende Kraft festgestellt werden kann. Dies ist durch folgende Einrichtung erzielt (s. Fig. 1).

Durch den Exzenter $E$, welcher entweder durch eine Kurbel mit der Hand oder durch einen Elektromotor mit Schnurlaufübertragung in Rotation versetzt werden kann, wird dem Stahlhebel $\boldsymbol{F}_{1} \boldsymbol{F}_{2}$ (Drehachse in $A$ ) eine bestimmte Bewegung aufgezwungen. Er wird gegen den Exzenter durch eine Spiralfeder angedrückt erhalten. Um die Reibung des Exzenters gegen den Hebel möglichst klein zu machen, ist an diesem, senkrecht unter der Achse des Exzenters, eine kleine in Spitzen laufende Rolle $R$ angebracht. Die Bewegungen des Stahlhebels werden nun einerseits durch einen in seiner Verlängerung angebrachten Strohhebel $H_{1}$ direkt registriert und andererseits auf die Trommel $T T$ übertragen. Diese hat eine lichte Weite von 25 und eine Höhe von $40 \mathrm{~mm}$. An beiden Grundflächen ist sie mit stark gespannten Gummimembranen $G_{1}$ und $G_{2}$ überzogen, welche in Nuten festgebunden sind. Gegen die Membranen werden Ringe $r r$ von versehiedenem Aussehnitt durch die Überwurfmuttern $m m$ angedrückt, so dass kreisförmige Ausschnitte der Gummimembranen von abstufbarer Grösse zutage treten. Zwei mit den Hähnen $H h_{1}$ und $H h_{2}$ verschliessbare Zuleitungsröhren ermöglichen die luftfreie Füllung der Trommel.

Die Bewegungen des Stahlbebels $F_{1} F_{2}$ werden auf die untere Gummimembran $G_{1}$ in folgender Weise übertragen: Auf die Membran

1) Neuerdings wieder von Athanasiu (Méthode graphique, trav. de l'assoc. de l'Institut Marey t. 1 p. 29. Paris 1905) zur Prüfung von Hebeln und Sphygmographen.

2) Frank, Prinzipien. Zeitschr. f. Biol. Bd. 45 S. 480. „Auch der grösste Teil der bisher vorgeschlagenen experimentellen Prüfungsmethoden, wie die Donders'sche oder die Buisson'sche, zur Prüung der Sphygmographen, ist entweder im Prinzip falsch oder ungenügend. Man muss genau die Konstanten der Instrumente kennen, welche für die richtige Darstellung der Bewegungen von Bedeutung sind." Ferner: Zeitschr. f. Biol. Bd. 59 S. 451. 
ist eine Messingplatte $\boldsymbol{P}$ aufgeklebt, aus deren Mitte sich ein stiftförmiger Fortsatz $\left(S t_{1}\right)$ erhebt; dieser wird mit einem entsprechenden, mit dem Stahlhebel vernieteten Stift $S t_{2}$ verschraubt, wobei der Abstand zwischen der Membran und dem Stahlhebel innerhalb gewisser Grenzen verändert werden kann. In allen Fällen ist die Platte $P$ und damit auch die Membran $G_{1} \mathrm{zwangsläufig} \mathrm{mit} \mathrm{dem} \mathrm{Stahl-}$ hebel verbunden ${ }^{1}$ ).

Die Einstellung der Platte $\boldsymbol{P}$ geschah jeweils derart, dass die Stifte $S t_{1}$ und $S t_{2}$ in der Phase der grössten Entfernung des Hebelarmes $F_{2}$ von der Membran $G_{1}$ versehraubt wurden; in dieser Lage wurde dann in der Trommel ein Überdruck von $10 \mathrm{~cm}$ Wasser erzeugt; es trat daher bei keiner Stellung des Exzenters ein negativer Druck in der Trommel auf.

Die der Membran $G_{1}$ mitgeteilten Bewegungen werden bei geschlossenen Hähnen ausschliesslich auf die Membran $G_{2}$ übertragen, deren Volumelastizität durch den verschiedenen Ausschnitt des Ringes $r r$ sowie durch verschiedene Dicke und Spannung der Membran geändert werden kann. Die Bewegungen dieser Membran können durch einen Hebel $H_{2}$ von geringem Trägheitsmoment gleichfalls registriert werden. An die Trommel $T T$ wird nun das zu prüfende Manometer $M$ durch undehnbare Verbindungen angeschlossen und das ganze System mit Wasser gefüllt. Bei meinen Versuchen war das Manometer in allen Fällen senkrecht über der Trommel und auf derselben Grundplatte mit dieser derart angebracht, dass die drei Hebelspitzen $H_{1}, H_{2}$ und $H_{3}$ eine senkrechte Linie bildeten. Dabei waren das Manometer und die Trommel $T$ durch eine U-förmig gebogene Glasröhre miteinander verbunden, welche an beiden Enden in kegelförmig auslaufende Messinghülsen gekittet und durch die Überwurfmuttern $U_{1}$ und $U_{2}$ an die beiden Körper angeschlossen war. Die Verbindungsröhre hatte bei den im folgenden

1) Diese Art der Verbindung war auch in dem früher von mir gebrauchten Prüfungsapparat (Pflüger's Arch. Bd. 55 S. 323) vorhanden; es ist daher der Satz, welchen Frank bei der Betrachtung der Rückwirkung zwischen verkoppelten Kräften (Zeitschr. f. Biol. Bd. 53 S. 447) ausspricht: „So hat Donders bei der Schaffung seiner Prüfungsmethode für den Sphygmographen nicht an sie gedacht, auch nicht Hürthle bei der Anwendung dieses Verfahrens", für die von mir ausgeführten Untersuchungen jedenfalls unbegründet und für die Donders'schen sehr wahrscheinlich gleichfalls, weil auch an dessen Apparat durch geeignete, von Donders ohne Zweifel beachtete Einstellung der von Frank vermutete Fehler vermieden wird. 
beschriebenen Versuchen eine Länge von $42 \mathrm{~cm}$ (vom Trommel- bis zum Manometerkörper gerechnet) und einen Durchmesser von $6,8 \mathrm{~mm}$ im Lichten; die wirksame Masse der Manometer war daher 115.

Der Gummimembran $G_{2}$ kommt eine doppelte Bedeutung zu:

1. als elastischer Faktor verhindert sie, dass die Bewegungen des Manometers zwangsläufige werden und bringt damit das Manometer unter Bedingungen, ähnlich denjenigen, wie sie im Kreislauf gegeben sind ${ }^{\text {) }}$;

2. dient die Membran zur Feststellung der Rückwirkung: Während nämlich bei der Ausschaltung des Manometers die Kurven der Hebel $H_{1}$ und $H_{2}$ übereinstimmen, werden nach der Einschaltung des Manometers durch Öffnung des Hahnes $H h_{2} \mathrm{Ab}$ weichungen am Hebel $H_{2}$ bemerkbar, als Zeichen der Rückwirkung des Manometers auf den Trommelinhalt. Diese kommt einerseits dadurch zustande, dass die Tätigkeit des Manometers durch eine Flüssigkeitsbewegung aus der Trommel nach dem Manometer unter* halten, der in der Trommel herrschende Druck also herabgesetzt wird; die Rückwirkung wird daher mit der am Manometer zu leistenden Arbeit wachsen. Andererseits beeinflussen aber auch die im Manometer und in der Zuleitungsröhre auftretenden Druckschwankungen, falls sie quantitativ oder im Phasenverlauf von den ursprünglichen abweichen, den Druckverlauf in der Trommel, indem sie mit ihm interferieren; die Kurve des Hebels 2 weicht in diesem Falle von der des Hebels 1 ab; der Apparat gibt daher ein Mittel an die Hand, nicht allein die Abweichungen der vom Manometer verzeichneten Kurve von der primären festzustellen (durch Vergleichung der Kurven der Hebel 1 und 3), sondern auch die Rückwirkung des Manometers auf die bewegende Kraft (durch Vergleichung der Kurven 2 und 1).

Die Prüfungen der Manometer verliefen in folgender Weise:

Der Exzenter wurde durch einen Elektromotor in Bewegung versetzt. Durch Transmissionsscheiben von versehiedenem Durchmesser konnten sechs verschiedene Geschwindigkeiten hergestellt werden. Sie ergaben mit kleinen Abweichungen 1,3-3,3 Um-

1) Bei dem älteren in Pflüger's Arch. Bd. 55 beschriebenen Modell war der zwischen Trommelrand und Grundplatte freibleibende ringförmige Ausschnitt der Gummimembran der einzige elastische Faktor. 
drehungen pro Sekunde. Es konnten daher die verschiedenen Manometer denselben Druckschwankungen ausgesetzt und ihre Leistungen unmittelbar verglichen werden.

Als darzustellende Bewegung wurde die trapezförmige Kurve des Druckablaufes in der Kammer gewählt, und zwar derart schematisiert, dass das systolische Plateau und die Pause als gerade Linien dargestellt und die mit den Verbindungslinien gebildeten Ecken abgerundet wurden (s. die Fig. a-c Taf. III).

Zur Prüfung des Apparates wurden zunächst die Kurven der Hebel 1 und 2 registriert, während das Manometer durch Schluss des Hahnes $H h_{2}$ ausgeschaltet war. Dabei ergab sich, dass die beiden Kurven nur bei der kleinsten. Ummdrehungszahl des Exzenters den reinen trapezförmigen Verlauf zeigten, bei den grösseren Geschwindigkeiten aber dureh Schwankungen in den horizontalen Teilen der Kurve, insbesondere in der Pause, entstellt wurden. Die Entstellungen waren gleichsinnig an den Kurven beider Hebel, nur stärker ausgeprägt an der des Hebels 2. Ihr unregelmässiger Verlauf macht es wahrseheinlich, dass es Erzitterungen sind, welche vom Elektromotor durch die Transmission auf den Exzenter übertragen wurden. Sie sind aus dem Grunde störend, weil sie die Feststellung der Rückwirkung des Manometers auf die Trommel $T$ erschweren. $\mathrm{Zu}$ ibrer Beseitigung müsste ein ruhiger Antrieb benutzt werden, der mir nicht zur Verfügung stand.

Nunmehr wurden die vier Russhebelmanometer gleicher Empfindlichkeit, aber von verschiedenem Membrandurchmesser an die Prüfungstrommel angeschlossen, welche in der vorhergehenden Abhandlung (Abschnitt G, S. 215) nach der Schwingungsmethode untersucht worden waren. Die Membrandurchmesser betrugen $0,54,0,72,0,9$ und $1,1 \mathrm{~cm}$. Ich bezeichne sie im folgenden als MI, II, III und IV, derart, dass MIV den grössten Membrandurchmesser hat. Jedes der Manometer wurde mit den sechs verfügbaren Umdrehungsgeschwindigkeiten (Pulszahlen) und zwar jeweils im ungedämpften. Zustand und mit den Dämpfungen von 0,6 und $0,4 \mathrm{~mm}$ geprüft. Man erhielt also von jedem Instrument 18 Pulsreihen. Bei diesen Prüfungen wurden nur die Kurven der Hebel $H_{2}$ und $H_{3}$ registriert, die des Hebels $H_{1}$ weggelassen, weil die Kurven $H_{1}$ und $H_{2}$ nur wenig Untersehied zeigten, und die oben erwähnten Erzitterungen eine genaue Feststellung der Rückwirkung störten.

Die bei den sechs verschiedenen Umdrehungsgeschwindigkeiten 
erzielten Ergebnisse sind grundsätzlich bei den vier Manometern übereinstimmend und nur quantitativ versehieden. Da die Unterschiede bei den grössten Leistungen am deutlichsten bervortreten, beschränke ich mich auf die Mitteilung der Resultate bei der grössten Umdrehungsgeschwindigkeit, welche $3,3-3,4$ Pulse pro Sekunde lieferte. Taf. III enthält Proben dieser Prüfung. Die unteren, mit $a, b$ und $c$ bezeichneten Kurvenreihen enthalten jeweils die Kurven des Trommelhebels $H_{2}$, die oberen, mit $a_{1} b_{1} c_{1}$ bezeichneten die gleichzeitig registrierten des Manometerhebels $H_{3}$. Die Kurven $a a_{1}$ sind jeweils bei ungedämpftem Manometer registriert; $b b_{1}$ nach Einschaltung der Dämpfung $0,6 \mathrm{~mm} ; c c_{1}$ bei Dämpfung $0,4 \mathrm{~mm}$.

Was zunächst die Frage betrifft, ob die vier zu vergleichenden Manometer wirklich von gleicher statischer Empfindlichkeit gewesen seien, so ergab die nach den einzelnen Versuchen vorgenommene Eichung, dass die Manometer III und IV ziemlich genau übereinstimmen, dass das Manometer II aber von höherer Empfindlichkeit, gegen die ersteren also im Nachteil war; umgekehrt war Manometer I von etwas geringerer Empfindlichkeit als die ersteren. Diese Erscheinung kommt auch in der Höhe der Kurven deutlich zum Ausdruck, wenn man nur die Kurvenhöhen bei der Dämpfung $0,4 \mathrm{~mm}$ vergleicht. Sie betragen 7,8, 7,9,9,0 und 6,8 mm. Die Kurvenböhen der ungerämpften Manometer lassen sich nicht ohne weiteres vergleichen, da sie durch Eigenschwingungen mehr oder weniger entstellt sind.

Was die Wiedergabe der Grundkurve anlangt, so zeigt sich bei allen Manometern die auffallende Erscheinung, dass die horizontalen Linien (Plateau und Pause) in der Weise unrichtig dargestellt werden, dass sie, von den Eigenschwingungen abgesehen, Winkel mit der Abszisse bilden. Um die Grösse dieser Abweichungen bei den einzelnen Instrumenten $\mathrm{zu}$ bestimmen, habe ich die Ordinaten am Anfang $\left(h_{a}\right)$ und Ende $\left(h_{c}\right)$ des Plateaus gemessen und aus beiden einen Quotienten $\frac{h_{a}}{h_{e}}$ gebildet, der ein Maß für die Grösse des Fehlers abgibt. Die Ordinaten sind in Spalte VI und VII der folgenden Tabelle, die Quotienten in Spalte VIII verzeichnet. Letztere zeigt nun, dass die Quotienten am ungedämpften Manometer mit dem Membrandurchmesser im allgemeinen kleiner werden, nur die grösste Membran macht eine Ausnahme. Ferner nehmen sie in allen Fällen mit der Dämpfung ab. Sie scheinen daher durch die Trägheit der Flüssigkeit veranlasst zu sein, doch ist der langsame Abfall der Linie nicht ganz klar. 
Die Prüfung der M anome er mit Druckschwankıngen von bekannter Form. 233

\begin{tabular}{|c|c|c|c|c|c|c|c|}
\hline $\mathrm{I}$ & II & III & IV & $\nabla$ & VI & VII & VIII \\
\hline \multirow{2}{*}{$\begin{array}{l}\text { Mano- } \\
\text { meter } \\
\mathrm{cm}\end{array}$} & \multirow{2}{*}{ Dämpfung } & \multirow{2}{*}{$\begin{array}{l}\text { Pulszahl } \\
\text { pro Sek. }\end{array}$} & \multicolumn{2}{|c|}{ Anstiegszeit des } & \multicolumn{3}{|c|}{ Sekundäre Pulse } \\
\hline & & & $\begin{array}{c}\text { Pulses II } \\
\text { Sek. }\end{array}$ & $\begin{array}{c}\text { Pulses III } \\
\text { Sek. }\end{array}$ & $h_{a}$ & $h_{e}$ & $\frac{h_{a}}{h_{\epsilon}}$ \\
\hline I $(0,5$ & $\begin{array}{c}\text { unged. } \\
0,6 \\
0,4\end{array}$ & $\begin{array}{l}3,4 \\
3,4 \\
3,4\end{array}$ & $\begin{array}{l}0,036 \\
0,038 \\
0,035\end{array}$ & $\begin{array}{l}0,030 \\
0,034 \\
0,041\end{array}$ & $\begin{array}{l}8,5 \\
8,5 \\
6,8\end{array}$ & $\begin{array}{l}7,0 \\
7,0 \\
6,7\end{array}$ & $\begin{array}{l}1,21 \\
1,21 \\
1,02\end{array}$ \\
\hline II $(0,7$ & $\begin{array}{c}\text { unged. } \\
0,6 \\
0,4\end{array}$ & $\begin{array}{l}3,4 \\
3,4 \\
3,3\end{array}$ & $\begin{array}{l}0,037 \\
0,036 \\
0,036\end{array}$ & $\begin{array}{l}0,037 \\
0,040 \\
0,054\end{array}$ & $\begin{array}{r}10,8 \\
9,8 \\
9,0\end{array}$ & $\begin{array}{l}8,4 \\
8,2 \\
8,2\end{array}$ & $\begin{array}{l}1,29 \\
1,20 \\
1,10\end{array}$ \\
\hline III $(0,90)$ & $\begin{array}{c}\text { unged. } \\
0,6 \\
0,4\end{array}$ & $\begin{array}{l}3,3 \\
3,3 \\
3,3\end{array}$ & $\begin{array}{l}0,041 \\
0,045 \\
0,044\end{array}$ & $\begin{array}{l}0,036 \\
0,050 \\
0,079\end{array}$ & $\begin{array}{r}11,6 \\
8,9 \\
8,0\end{array}$ & $\begin{array}{l}7,8 \\
7,7 \\
7,8\end{array}$ & $\begin{array}{l}1,49 \\
1,16 \\
1,02\end{array}$ \\
\hline IV $(1,11)$ & $\begin{array}{c}\text { unged. } \\
0,6 \\
0,4\end{array}$ & $\begin{array}{l}3,4 \\
3,3 \\
3,4\end{array}$ & $\begin{array}{l}0,042 \\
0,037 \\
0,039\end{array}$ & $\begin{array}{l}0,038 \\
0,057 \\
0,102\end{array}$ & $\begin{array}{r}12,2 \\
8,7 \\
7,8\end{array}$ & $\begin{array}{c}9,2 \\
7,9 \\
\text { kein Plateau }\end{array}$ & $\begin{array}{l}1,33 \\
1,10 \\
-\end{array}$ \\
\hline
\end{tabular}

Die Werte der Anstiegszeit sind in Spalte IV und V der Tabelle enthalten und zwar von den Kurven der Hebel 2 und 3. Da die Pulszablen in allen Fällen annähernd gleich sind, ist zu erwarten, dass die Anstiegszeiten des Hebels 2 in allen Fällen gleich sind. Das ist denn auch bis auf kleine Abweichungen der Fall, die wohl als Messungsfehler oder Unregelmässigkeiten in der Rotation des Apparates aufzufassen sind.

Die Anstiegszeiten der Manometerkurven sind bei ungedämpften Manometern in allen Fällen gleich, dagegen zeigen sich, wie zu erwarten ist, nach Einschaltung der künstlichen Dämpfungen wesentliche Unterschiede in dem Sinne, dass die Anstiegszeiten der Manometer um so mehr verlängert werden, je grösser die Membrandurchmesser sind. Die Manometer gleicher Empfindlichkeit stehen also, wenn man ihre Leistungsfähigkeit nach der Anstiegszeit bei gleicher Dämpfung beurteilt, in umgekehrter Reihenfolge wie die Membrandurchmesser.

Was die Wiedergabe der geforderten Kurven betrifft, so genügt keines der vier Instrumente im ungedämpften Zustand zur einwandfreien Darstellung; dabei ist zu berücksichtigen, dass die Darstellung von 200 Pulsen der vorliegenden Form pro Minute auch eine Anforderung ist, wie sie kaum von einem Manometer bei physiologischen Versuchen verlangt werden wird. Ein Blick auf die Kurven der Taf. III zeigt aber, dass die Entstellung wiederum mit dem Membrandurchmesser zunimmt. Beim Manometer I besteht sie aus einer kleinen Schleuderung am Ende des Aufstiegs und Abstiegs 
und im schrägen Verlauf der horizontalen Linien. Bei den andern Manometern treten immer stärker ausgeprägte Eigenschwingungen im Plateau und insbesondere in der Pause auf.

Durch Einschaltung der Dämpfung 0,6 mm werden diese Eigenschwingungen grossenteils beseitigt, und die Kurven der vier Manometer werden sich ähnlicher. Durch die Dämpfung $0,4 \mathrm{~mm}$ endlich werden die Kurven der Manometer I und II noch weiter verbessert, die der beiden anderen aber versehlechtert: bei der Kurve des Manometers IV verschwindet das Plateau und sie nimmt die Form einer eingipfligen Kurve mit abgerundeter Spitze an, unter beträchtlicher Verlängerung der Anstiegszeit, desgleichen verschwindet der scharfe Übergang des absteigenden Schenkels in den horizontalen. - Ähnliches, nur weniger stark ausgebildet, bemerkt man beim Manometer III; ein Plateau ist noch vorhanden, aber etwra auf die Hälfte gekürzt. - Wesentlich besser sind die Kurven des Manometers II; doch fehlt auch hier der scharfe Übergang des aufsteigenden Schenkels in das Plateau und namentlich der des absteigenden Schenkels in die Linie der Pause. - Die beste Darstellung gibt ohne Zweifel Manometer IV mit der kleinsten Membran. Sie kann als getreue Wiedergabe des geforderten Pulses betrachtet werden, mit Ausnahme des allmählichen Überganges des absteigenden Schenkels in den horizontalen.

Was schliesslich die Rückwirkung des Manometers auf die Trommel $T T$ betrifft, so ist deren quantitative Feststellung durch die obengenannten Erzitterungen etwas erschwert, doch lässt sich das Folgende aus den Kurven mit Sicherheit entnehmen:

Eine Rückwirkung des Manometers auf die Trommel ist nur am ungedämpften Manometer zu erkennen, und zwar in Form von Druckschwankungen an der horizontalen Linie während der Pause; am gedämpften ist eine deutliche Rückwirkung nicht nachzuweisen. Von den einzelnen Manometern übt das Manometer IV entschieden die grösste Rückwirkung aus, wie von vornherein zu erwarten ist, und wie ein Blick auf die Kurven der einzelnen Manometer zeigt.

Vergleicht man das vorliegende Ergebnis mit demjenigen, welches dieselben Manometer, nach der Schwingungsmethode untersucht, geliefert haben, so stimmen beide im allgemeinen überein, nur beim Man. I $(0,54 \mathrm{~cm}$ Membran) gehen sie auseinander: nach der Schwingungsmethode untersucht zeigt dieses nur dann die höhere Schwingungszahl im Vergleich zu den Manometern mit grösserer 
Membran, wenn die wirksame Masse relativ gross (550) ist, während bei kleiner wirksamer Masse (100) das Man. II (0,72 cm Membran) die höchste Schwingungszahl zeigt (Tab. XXVIc S. 220 der vorhergehenden Abhandlung). Da nun bei der Prüfung mit künstlichen Druckschwankungen die wirksame Masse 115 ist (s. S. 230), so wäre nach der Schwingungsmethode zu erwarten gewesen, dass die Leistungen des Man. II die höchsten sind. Das ist aber nicht der Fall; es wird vom Man. I entschieden übertroffen.

Ich bin daher nicht überzeugt, dass die Schwingungszahl einen in allen Fällen zuverlässigen $M a ß s t a b$ für die Leistungen der Manometer beim praktischen Gebrauch darstellt, sondern halte eine weitere vergleichende Prüfung der In-

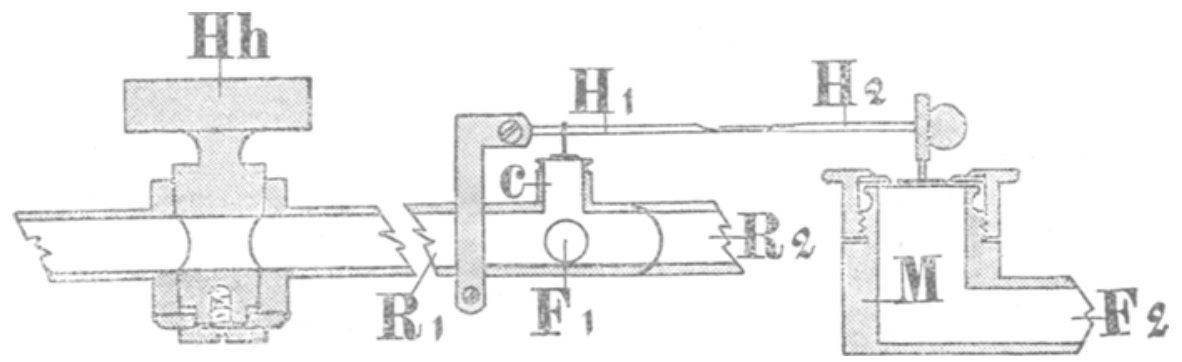

Fig. 2.

strumente nach beiden Methoden zur Entscheidung der Frage für notwendig. Jedenfalls geht aber auch aus dieser Prüfung in überzeugendier Weise hervor, wie ungerechtfertigt das Urteil Frank's über den Membranquerschnitt meiner Hebelmanometer ist.

Versuchsweise habe ich zur Prüfung der Manometer mit künstlichen Druckschwankungen noch eine $z$ weite Methode benutzt, welche gegenüber der eben beschriebenen gewisse Vorzüge aber auch Nachteile hat. Sie besteht darin, dass Druckschwankungen in einer strömenden Flüssigkeit erzeugt und einerseits direkt, andererseits von dem zu prüfenden Manometer registriert werden. Zur Erzeugung der Druckschwankungen benutzte ich die rhythmische Öffnung und Schliessung des Hahnes $H h$, Fig. 2; er mündet in den am Boden befindlichen Tubulus einer wassergefüllten Flasehe in welcher ein Druck von etwa $150 \mathrm{~cm}$ herrscht. An den Hahn ist die Röhre $R_{1} R_{2}$ von $9 \mathrm{~mm}$ Durchmesser durch ein mehr oder weniger dehnbares Gummistück angeschlossen und an $R_{2}$ wieder eine in der Figur nicht gezeich- 
nete elastische oder starre Röhre, welche an ihrem freien Ende einen durch einen Quetschhahn regulierbaren Widerstand bat. Bei der rhythmischen Drehung des Habnes $H h$ entsteht nun eine pulsatorische Strömung durch das Röhrensystem, deren Geschwindigkeit durch den regulierbaren Widerstand abgeändert werden kann, und deren Nebenwellen um so rascher verlaufen, je kürzer das System und je grösser seine Elastizität ist. Sind die Röhren aus Glas oder Metall und durch kurze, wenig dehnbare Gummistücke verbunden, so erhält man rasch verlaufende Druckschwankungen mit komplizierten Nebenwellen, deren Darstellung grosse Anforderungen an ein Manometer stellt.

Die in der Röhre $R_{1} R_{2}$ auftretenden Druckschwankungen werden in folgender Weise direkt registriert: Auf die Röhre ist der kurze Zylinder $C$ von $5 \mathrm{~mm}$ lichter Weite und gleicher Höhe aufgesetzt, der mit einer starken, straff gespannten Gummimembran überzogen wird. Die dieser Membran mitgeteilten Bewegungen werden durch einen Hebel $H_{1}$ von sehr geringem Trägheitsmoment zunächst mechanisch und dann optisch vergrössert registriert.

Unterhalb des Zylinders $C$ und senkrecht zu diesem zweigt aus der Röhre $R_{1} R_{2}$ ein Fortsatz $F$ von $7 \mathrm{~mm}$ Durchmesser und $3 \mathrm{~cm}$ Länge ab, der mit der Zuleitungsröhre $F_{2}$ des Manometers $M$ durch Röhren vou verschiedenen Dimensionen verbunden werden kann. Nacbdem das ganze System luftfrei mit Wasser gefült ist, zeichnen die beiden Hebel $H_{1}$ und $H_{2}$ bei den Drehungen des Hahnes $H h$ ihre Kurven unmittelbar übereinander auf und gestatten so eine direkte Vergleichung.

Bei der Diskussion dieser Methorle ist zu bedenken, dass die vom Manometer darzustellenden Druckschwankungen zunächst nicht von bekannter Form sind wie bei der vorhergehenden. - Die Druckschwankung muss vielmehr erst durch den Registrierapparat $C H_{1}$ dargestellt werden, und es fragt sich, ob sie durch diesen unentstellt wiedergegeben wird. Dies kann unbedingt angenommen werden, denn die wirksame Masse ist praktisch gleich Null (in Wirklichkeit 2,54), $E^{\prime}$ sehr gross, schätzungsweise $100 \cdot 10^{6}$. Es könnte also nur die wirksame Masse des Hebels $H_{1}$ störend wirken. Gibt man aber diesem ein sehr geringes Trägheitsmoment und wendet nicht mehr als sechsfache Hebelverorösserung an, so darf man zuversichtlich erwarten, dass die von $H_{1}$ registrierten Bewegungen 
Die Prüfung der Manometer mit Druckschwankungen von bekannter Form. 237 mit den bei Ausschluss von $C$ vorhandenen identisch sind. Wollte man die Bewegungen des Hebels $H_{1}$ aber nicht optisch, sondern mechanisch registrieren, dann müsste man sich allerdings durch besondere Versuche überzeugen, ob die erhaltenen Kurven den Druckverlauf in der Röhre unentstellt wiedergeben.

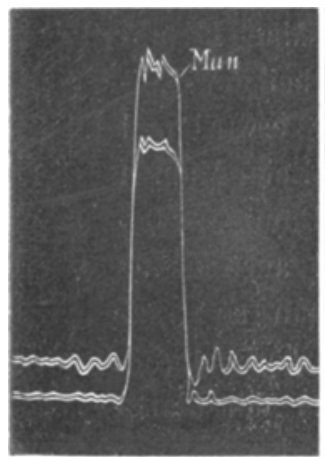

Man. II a, ungedämpft.

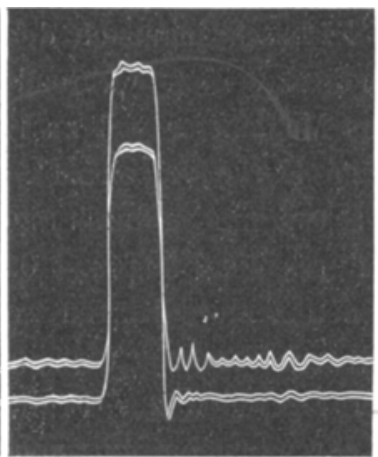

Man. II b, Dampfung 0,6 .

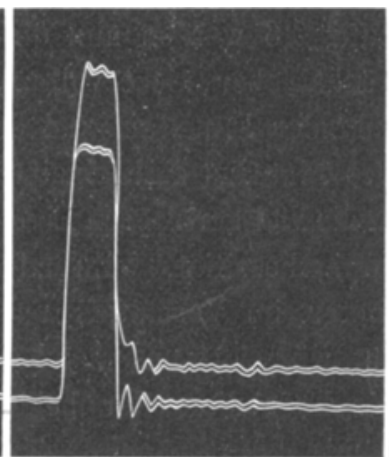

Man. II c, Dãmpfung 0,4.

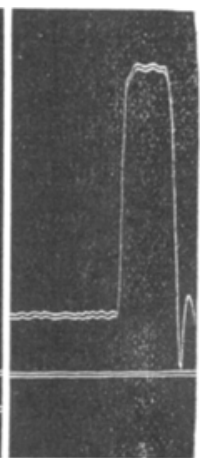

Man. II d.

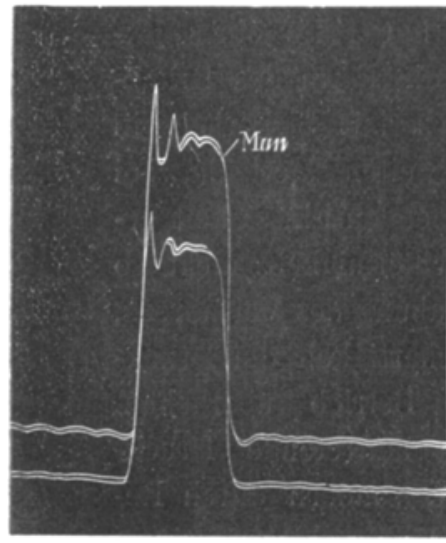

Man. IV a, ungedämpft.

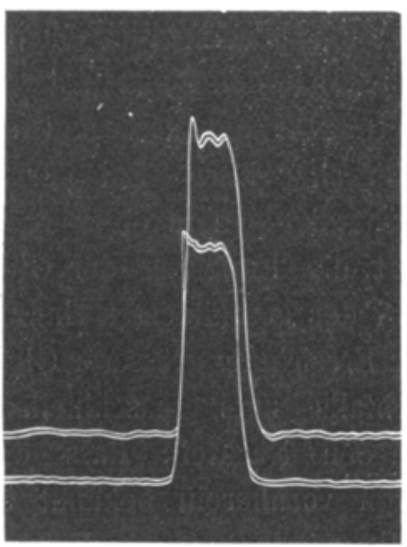

Man. IV b, Dămpfung 0,6 .

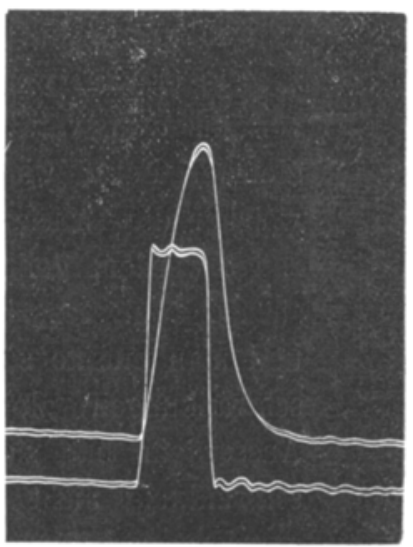

Man. IV c, Dãmpfung 0,4.

Fig. 3.

Mit dieser Methode wurde vorläufig eine vergleichende Prüfung der oben beschriebenen Manometer II und IV ${ }^{1}$ ) (mit 0,72 und $1,11 \mathrm{~cm}$ Membrandurchmesser) im gedämpften und ungedämpften Zustand vorgenommen. Das Ergebnis ist aus den Kurven der Fig. 3 za ersehen. Die Kurven des Hebels 1 liegen in allen Fällen unten, die des Manometerhebels oben. Die Aussebläge des Manometerhebels sind etwas grösser als die des Hebels 1.

1) Experimentalkritik S. 216. 
Im ungedämpften Zustand wiederholen beide Manometer die Kurven des Hebels 1. Allein bei der Ausschaltung der Manometer [Kurve IId] ${ }^{1}$ ) oder schon bei der Einschaltung der Dämpfungen zeigt sich, dass die ursprüngliche Druckschwankung durch die Rückwirkung der Manometer entstellt wird. Diese Entstellung ist aber wesentlich grösser beim Man. IV als beim Man. II. Durch die Dämpfung 0,6 werden nun die Eigenschwingungen des Man. IV abgeschwächt, aber nicht vollständig beseitigt, während bei der Einschaltung der Dämpfung 0,4 das Manometer viel zu träge wird und statt des Plateaus eine eingipflige Kurve verzeichnet. Beim Man. II werden die viel geringeren Eigenschwingungen durch die Dämpfung 0,6 vollständig beseitigt, während durch die Dämpfung 0,4 auch dieses Manometer im Verhältnis zu den Anforderungen sehon so träge gemacht wird, dass es das Plateau nicht ganz ungekürzt wiedergibt.

Die Ergebnisse der beiden Methoden zur Prüfung der Manometer mit künstlichen Druckschwankungen stimmen also in den wesentlichen Punkten überein. Die erste, bei welcher die Druckschwankungen in einer ruhenden Flüssigkeit mit Hilfe eines Exzenters erzeugt werden, hat den Vorteil, dass die Form des Druckverlaufs von vornherein bekannt ist und sich mit Sicherheit in gleicher Form wiederholen lässt. Bei der zweiten Methode, bei welcher die Druckschwankungen in einer strömenden Flüssigkeit erzeugt werden, gelingt die Erzielung der Gleichförmigkeit nicht so sicher, wenigstens bei der geschilderten Handhabung des Verfahrens, und man müsste zur Erreichung grösserer Gleichartigkeit der Druckschwankungen gleichfalls zum maschinellen Betrieb übergehen. Ausserdem hat sie den Nachteil, dass die auftretende Druckschwankung nicht von vornherein bekannt ist, sondern durch Registrierung festgestellt werden muss. Einen Vorzug der Methode sebe ich in dem Umstand, dass sie das Manometer unter Bedingungen prüft, welche den im Kreislauf vorbandenen ähnlicher sind als die der ersten Methode. Möglicherweise lassen sich die angegebenen Nachteile künftighin dadurch beseitigen, dass man beide Methoden kombiniert, d. h. Druckschwankungen in einer strömenden Flüssigkeit mit Hilfe eines Exzenters herstellt.

1) Die Kurve des Hebels 1 nach Ausschaltung des Manometers IV ist nicht wiedergegeben, da sie mit der Kurve des Hebels 1 bei Einschaltung der Dämpfung 0,4 übereinstimmt. 
Die Prüfung der Manometer mit Druckschwankungen von bekannter Form. 239

\section{Zusammenfassung der Ergebnisse.}

Da die Korrektur der vom Manometer verzeichneten Kurven auf Grund der Theorie mit Hilfe der Zahl der Eigenschwingungen und der Dekremente die eingangs genannten Fehler hat, und da nicht erwiesen ist, dass die Schwingungszahl des Manometers auch in den Fällen ein Maßstab seiner Güte ist, in welchen die Theorie versagt, ist es wichtig, ein allgemein gültiges Verfahren zur Prüfung der Leistungen der Manometer zu besitzen. Ein solches ist im Vorhergehenden in zwei Modifikationen beschrieben und hat zu folgendem Ergebnis geführt:

1. Die Leistungen der Hebelmanometer gleicher Empfindlichkeit wachsen mit abnehmendem Membranquerschnitt bis herab zu $5^{1 / 2} \mathrm{~mm}$ Durchmesser.

2. Durch geeignete Dämpfung wird die Leistung der Manometer erhöht.

3. Mit Hilfe der beschriebenen Methode lässt sich auch die Rückwirkung des Manometers auf die einwirkende Kraft nachweisen.

4. Die Prüfung der Manometer mit künstlichen Druckschwankungen von bekannter Form führt nicht durchweg zu denselben Ergebnissen wie die Prüfung nach der Sehwingungsmethode. 